Introduction There are no agreed endoscopic signs for the diagnosis of villous atrophy(VA) in coeliac disease(CD), necessitating biopsies and for both diagnosis and exclusion. Here we evaluated the role of near focus Narrow Band Imaging (NF-NBI) for the assessment of villous architecture in suspected $\mathrm{CD}$ with development and further validation of a novel NF-NBI classification.

Methods Patients with symptoms/investigations warranting duodenal biopsy were prospectively recruited between September 2017 to August 2018. Six paired NF-white light(NFWLE) and NF-NBI images with biopsy (2 from the first part of the duodenum,4 from the second) were obtained from each patient. Histopathology grading used Marsh-Oberhuber classification(M-O). Images were reviewed for quality and biopsy orientation. Separate images were used for development of the classification, training and validation steps. A modified Delphi process was performed on images and video recordings by 3 endoscopists to define NF-NBI characteristics (included if kappa>0.6). 13 blinded endoscopists(5 expert, 8 non-expert) underwent a short training module on the proposed NBI classification and evaluated paired(NF-WLE/NFNBI) images.

Results 100 consecutive patients were recruited and $n=97$ completed the study $(66 \mathrm{~F}, 51.2 \pm 17.3 \mathrm{yrs})$. TTG positive $\mathrm{n}=17 / 89$. Prevalence of $\mathrm{M}-\mathrm{O} \mathrm{VA}(3 \mathrm{a} / 3 \mathrm{~b} / 3 \mathrm{c})$ in D1 and D2 biopsies was 52/194(27\%) and 70/388(18\%) respectively. After image quality and biopsy orientation review; 548 paired images remained. 498 paired images developed the classification; 3 descriptors:Villous shape, vascular discrimination, crypt phenotype proposed the classification. 13 endoscopists evaluated 50 paired images each(D1:20, M-O $0: 26$, M-O 3a-3b:13, M-O 3c:11). Pooled diagnostic test summary statistics(\%) for NF-NBI diagnosis of VA(Subtotal/ total atrophy) were: Sensitivity 97.9(91.67-100), specificity 82.15(62.5-100), NPV 97.7(92.59-100) and accuracy 89.7 (80-96) respectively. Mean difference in confidence using NF-NBI vs NF-WLE significantly improved when assessing the first part duodenum: The classification was further validated in histopathologically proven duodenitis $(n=15)$ images with no features of VA using the proposed classification.

Conclusion A novel NF-NBI classification for VA had been validated to reliably diagnose VA in suspected CD amongst both expert/non-expert endoscopists using readily available equipment and required only short training supporting translation to wider practice.

\section{AWE-05 CASE SERIES OF PER-ORAL ENDOSCOPIC PYLOROMYOTOMY IN PATIENTS WITH REFRACTORY GASTROPARESIS: A SINGLE CENTRE EXPERIENCE}

Nishmi Gunasingam*, Mehul Patel, Shradda Gulati, Amyn Haji, Bu'Hussain Hayee. King's College Hospital NHS Foundation Trust, London, UK

\subsection{6/gutjnl-2019-BSGAbstracts.23}

Introduction Gastroparesis (GP) is a disorder evidenced by delayed gastric emptying in the absence of mechanical obstruction, commonly idiopathic or secondary to diabetes mellitus. G-POEM has been used to treat refractory gastroparesis and we present our initial experience with this novel endoscopic technique.
Methods All patients undergoing G-POEM for refractory GP from May 2018 onwards were included, with data extracted from the hospital electronic patient record. Procedures were performed by one endoscopists at our centre with experience in submucosal endoscopy. Efficacy at 3 months was assessed by reduction of symptom score (Gastroparesis Cardinal Symptom Index; GCSI), with secondary considerations including technical success, procedural complications, hospital length of stay and hospitalisations after treatment.

Results 10 patients (10F; mean age $40.3 \pm 15.4$ y) were included (5 diabetic, 5 idiopathic). Mean duration of disease was $9.5 \pm 5.6 \mathrm{y}$, with 4 months median duration of follow-up post-procedure. 6 patients had previous treatment with botulinum toxin, and one gastric electrical stimulator. Technical success was achieved in all cases while, at 3 months, 8 had improvement total GCSI (mean scores 3.978 vs 2.076 $(\mathrm{p}=0.008))$ and each GCSI subtype score: nausea and vomiting $(3.63$ vs $1.70, \mathrm{p}=0.012)$; fullness $(4.30$ vs 2.30 , $\mathrm{p}=0.012$ ); bloating (4 vs $2.22, \mathrm{p}=0.28$ ). Mean hospital stay was $9 \pm 13 \mathrm{~d}$, with 5 patients staying less than 4 days and two with prolonged admission. One significant adverse event was recorded: abdominal collection around the myotomy site requiring a prolonged hospital admission of 18 days for intravenous antibiotics in one patient.

Only one patient required hospitalisation for gastroparesis symptoms after their G-POEM procedure (this was the same patient who did not achieve clinical success with reduction in their GCSI scores).

Conclusion G-POEM is a promising therapeutic treatment for patients with refractory GP, with significant improvement in symptoms and, in our cohort a dramatic reduction in the need for hospitalisation in short-term follow up. A European sham-controlled study is under way and longer-term data are required to confidently determine its role in the management of such a challenging condition.

\section{AWE-06 OUTCOMES OF HEMOSPRAY USE IN TUMOUR RELATED UPPER GASTROINTESTINAL BLEEDS: OUTCOMES FROM THE HEMOSPRAY REGISTRY}

${ }^{1}$ Mohamed Hussein*, ${ }^{2}$ Durayd Alzoubaidi, ${ }^{3}$ Miguel Fraile, ${ }^{3}$ Jacobo Ortiz Fernandez-sordo ${ }^{3}$ Krish Ragunath, ${ }^{4}$ Radu Rusu, ${ }^{4}$ Jason Dunn, ${ }^{14}$ Johannes Rey, ${ }^{5}$ Shraddha Gulati, ${ }^{5}$ Bu'Hussain Hayee, ${ }^{6}$ Selena Dixon, ${ }^{6}$ Sulleman Moreea, ${ }^{7}$ Duncan Napier, ${ }^{7}$ John Anderson, ${ }^{8}$ Martin Dahan, ${ }^{9}$ Max Hu, ${ }^{9}$ Patricia Duarte, ${ }^{9}$ Phil Boger, ${ }^{10}$ John Mcgoran, ${ }^{10}$ Inder Mainie, ${ }^{11}$ Alberto Murino, ${ }^{11}$ Sina Jameie-oskooei, ${ }^{11}$ Edward Despott, ${ }^{12}$ Cora Steinheber, ${ }^{12}$ Martin Goetz, ${ }^{13}$ Sharmila Subramaniam, ${ }^{13}$ Pradeep Bhandari, ${ }^{1,2}$ Laurence Lovat, ${ }^{8}$ Emmanuel Coron, ${ }^{14}$ Ralf Kiesslich, ${ }^{1}$ Rehan Haidry. ${ }^{1} U C L H, U K ;{ }^{2} U C L, U K ;{ }^{3}$ Nottingham Digestive Diseases Centre, UK; ${ }^{4}$ Guys and St Thomas Hospitals, UK; ${ }^{5}$ Kings college Hospital, UK; ${ }^{6}$ Bradford Teaching Hospital NHS Trust, UK; ${ }^{7}$ Gloustershire Hospitals NHS Foundation Trust, UK; ${ }^{8}$ University Hospital of Nantes, France; ${ }^{9}$ University Hospital Southampton, UK; ${ }^{10}$ Belfast NHS Trust, UK; ${ }^{11}$ Royal Free Hospital, UK; ${ }^{12}$ Tübingen University Hospital, UK; ${ }^{13}$ University of Portsmouth, UK; ${ }^{14}$ Horst Schmidt Kliniken, UK

\subsection{6/gutjnl-2019-BSGAbstracts.24}

Introduction Patients with tumour related upper gastrointestinal bleeds (UGIBs) are challenging to treat. Hemospray (Cook Medical, North Carolina, USA) is a novel haemostatic powder for GI bleeding. The primary aim was to look at outcomes of UGIBs secondary to tumours who had Hemospray therapy in 13 centres.

Methods Data was prospectively collected on the use of hemospray from specialist centres in the UK, France, Germany (Jan'16-September'18). Hemospray was used for UGIBs 
Abstract AWE-06 Table 1

\begin{tabular}{|c|c|c|c|c|}
\hline & & Monotherapy $(n=51)$ & Combination therapy $(n=17)$ & Rescue Therapy $(n=7)$ \\
\hline \multirow[t]{2}{*}{ Blatchford } & Median & 10 & 9 & 11 \\
\hline & IQR & $7-12$ & $7-14$ & $10-13$ \\
\hline \multirow[t]{2}{*}{ Rockall } & Median & 8 & 8 & 7 \\
\hline & $\mathrm{IQR}$ & $7-9$ & $7-9$ & $4-7$ \\
\hline \multicolumn{2}{|c|}{ Haemostasis achieved } & $51 / 51(100 \%)$ & $15 / 17(88 \%)$ & $7 / 7(100 \%)$ \\
\hline \multicolumn{5}{|c|}{ Rockall 7 and 8: Predicted rebleed rate: $25-40 \%$} \\
\hline \multicolumn{2}{|l|}{ Re-bleed } & $4 / 44(9 \%)$ & $2 / 14(14 \%)$ & $1 / 7(14 \%)$ \\
\hline \multicolumn{5}{|c|}{ Rockall 7 predicted mortality rate: $20-30 \%$} \\
\hline \multicolumn{5}{|c|}{ Rockall 8 predicted mortality rate: $40-45 \%$} \\
\hline \multicolumn{2}{|c|}{ All cause 7-day mortality } & $2 / 44(5 \%)$ & $0 / 14$ & $0 / 7$ \\
\hline \multicolumn{2}{|c|}{ All cause 30-day mortality } & $9 / 44(21 \%)$ & $3 / 14(21 \%)$ & $0 / 7$ \\
\hline
\end{tabular}

secondary to tumours as a monotherapy, dual-therapy with standard haemostatic techniques or rescue therapy. Haemostasis was defined as the cessation of bleeding within 5 minutes of the application of hemospray.

Results 75 patients with tumoural UGIBs were recruited (51 males, 24 females, 22/75 (29\%) oesophageal, 49/75 (65\%) gastric, $4 / 75$ (5\%) doudenal). The median rockall score was 8 (IQR,7-9).

Immediate haemostasis was achieved in $73 / 75$ (97\%) of patients, 7/65 (11\%) had a rebleed, 12/65 (18\%) died within 30 days (all-cause mortality). Based on the baseline average total rockall score, the expected rebleed rate is $25-40 \%$, and expected mortality rate was $40-45 \%$ in our cohort.

$100 \%$ immediate haemostasis was achieved in duodenal and gastric tumours, and $91 \%$ in oesophageal tumours.

Outcomes with mono/combination therapy and rescue therapy (table1)

Conclusions Hemospray is effective for achieving immediate haemostasis in UGIBs secondary to upper GI tumours, which are considered difficult to treat.

When considering average rockall score the rebleed and mortality rate is better than predicted rates. Haemostasis is achieved in the majority allowing for patient stabilization and providing time for surgery/radiotherapy.

\section{AWE-07 APOLLO OVERSTITCH DEVICE FOR ENDOSCOPIC REVISION OF ROUX-EN-Y GASTRIC-BYPASS, UPDATES FROM THE LARGEST UK SERIES}

Ahmed Ghanem, Vasha Kaur*, Marta Mano Lopes, Jonathan Cousins, Ahmed Ahmed, Dav Bansi. St. Mary's Hosptal, Imperial College Healthcare NHS trust, London, UK

\subsection{6/gutjnl-2019-BSGAbstracts.25}

Introduction The Apollo OverStitch is a minimally invasive endoscopic suturing device which allows full thickness suturing without the need for surgery

Roux-en-Y gastric bypass (RYGB) can achieve up to $60 \%$ weight loss 2 years after surgery but $30 \%$ of patients will regain their weight within 2 years. The options for this group of patients are limited; redo surgery can be challenging with a greater risk of complications. Endoscopic revision of the gastro-jejunal anastomosis using the Apollo OverStitch device now offers an alternative option in these patients to achieve further weight loss. Here we report our experience using this device, which is the largest patient cohort in the UK to date.

Method Between April 2017 and December 2018, we have used the Apollo OverStitch device in 23 patients who had regained weight after an initial RVGB. All patients were discussed initially at our bariatric MDT. All patients underwent a prior gastroscopy to ensure a stoma size of at least $2 \mathrm{~cm}$. All cases were done under general anaesthetic.

Results Here we report the follow up data for our cohort. 91\% of patients were females. Mean weight loss at early follow up (mean 82 days) was $6.7 \%$ and at late follow up (mean 342 days) was 9.9\%. One patient had a re-do procedure, having dropped from $104 \mathrm{~kg}$ to $95 \mathrm{~kg}$ and then further to $88.8 \mathrm{~kg}$. There were no procedure related complications.

Conclusion Endoscopic revision of the RYGB stoma using the Apollo OverStitch device is an effective method of achieving further weight loss in these patients. The effects are sustained at 1 year. We are now developing our technique to incorporate more bites for each suture placed, as well as following the first line of sutures with a second in order to achieve a tighter effect on reducing the stoma size. We have done a further 7 patients since, making our cohort the largest in the UK. We propose that this management option be considered in all patients with weight regain after RYGB and a gastrojejunal stoma $>2 \mathrm{~cm}$ at gastroscopy.

\section{Posters}

\section{PTH-001 SEMS IS A RISK FACTOR FOR POST-ERCP PANCREATITIS: EXPERIENCE FROM A HIGH-VOLUME UK HOSPITAL} 1,2Alaa Abdelwareth*, 'Ravi Madhotra. ${ }^{1}$ Milton Keynes University Hospital, Milton Keynes,
UK; ${ }^{2}$ Assiut University Hospital, Egypt

\subsection{6/gutjnl-2019-BSGAbstracts.26}

Introduction Studies have attempted to examine the risk of post-ERCP pancreatitis (PEP) with the placement of biliary self-exapandable metal stent (SEMS) with conflicting results. The aim of this study is to investigate whether biliary SEMS is a potential risk factor for PEP.

Methods All patients who underwent ERCP between January 2016 and May 2017 at Gastrointestinal Endoscopy Unit at 